\title{
The Sudden Drop in Spectral Entropy Associated with Acute Hypercarbia during Robot-assisted Laparoscopic Prostatectomy
}

Youngrok Kim, Mi Hwa Chung, Eun Mi Choi, In-Jung Jun*

Department of Anesthesiology and Pain Medicine, Kangnam Sacred Heart Hospital, Hallym University College of Medicine, Seoul, Korea

DOI: $10.36347 /$ sjmcr.2020.v08i05.011

| Received: 06.05.2020 | Accepted: 14.05.2020 | Published: 19.05.2020

*Corresponding author: In-Jung Jun

Abstract

Case Report

Severe hypercapnia during surgery is associated with electroencephalographic changes. This report presents a case of a sudden drop in spectral entropy in response to severe hypercapnia during robot-assisted laparoscopic prostatectomy (RALP) in a 75-year-old patient. One hour after pneumoperitoneum and Trendelenburg position application, inadvertent subcutaneous emphysema occurred. Arterial $\mathrm{CO}_{2}$ partial pressure $\left(\mathrm{PaCO}_{2}\right)$ rose to $85 \mathrm{mmHg}$, severe respiratory acidosis ensued, and the entropy level dropped to 2. Subsequently, intra-abdominal pressure was reduced by lowering the angle of the Trendelenburg position. Hyperventilation improved respiratory acidosis. Termination of the operation immediately improved acidosis and the entropy level. The sudden drop in spectral entropy may be associated with the narcotic properties of carbon dioxide $\left(\mathrm{CO}_{2}\right)$.

Keywords: Entropy, Subcutaneous emphysema, Hypercapnia, Respiratory acidosis, Robot-assisted laparoscopic prostatectomy.

Copyright @ 2020: This is an open-access article distributed under the terms of the Creative Commons Attribution license which permits unrestricted use, distribution, and reproduction in any medium for non-commercial use (NonCommercial, or CC-BY-NC) provided the original author and source are credited.

\section{INTRODUCTION}

Recently, robot-assisted surgeries have become popular due to the minimal invasiveness of the procedure and the precise control of the surgical instruments that the assistance allows for [1]. However, to get a better surgical view, $\mathrm{CO}_{2}$ pneumoperitoneum with a steep Trendelenburg position is needed during robot-assisted laparoscopic prostatectomy (RALP) which results in significant changes in respiratory physiology [2]. $\mathrm{CO}_{2}$ pneumoperitoneum may be associated with pulmonary atelectasis [3]. Prolonged maintenance of a steep Trendelenburg position reduces functional residual capacity, increases airway pressure, increases $\mathrm{CO}_{2}$ absorption from the peritoneum, and disturbs diaphragm function [4]. These changes may result in an increase in end-tidal $\mathrm{CO}_{2}$ partial pressure $\left(\mathrm{PetCO}_{2}\right)$. Rarely, inadvertent subcutaneous emphysema may aggravate hypercarbia and cause respiratory acidosis $[3,4]$.

Spectral entropy is used to assess the depth of anesthesia. Besides the concentration of anesthetic agents, other factors, such as low body temperature, low blood pressure, and brain injury, may depress the entropy level $[5,6]$. Hypercapnia may also depress the entropy level, though the mechanism by which this would occur is not well understood [7]. This report presents a case of a sudden drop in entropy due to severe hypercapnia during RALP.

\section{CASE REPORT}

A 75-year-old, $176 \mathrm{~cm}, 58.9 \mathrm{~kg}$ man was scheduled for RALP. He had no underlying medical diseases and had received cervical spine operation three years prior due to cervical spinal disc herniation. No other problems were revealed during the preoperative workup. Upon arrival in the operating room, standard anesthesia monitoring and spectral entropy began. Anesthesia was induced with intravenous propofol and rocuronium. Intubation was facilitated with video laryngoscopy because the patient could barely extend his neck. Anesthesia was maintained with remifentanil and sevoflurane. Adequate depth of anesthesia was maintained with spectral entropy of 40-60. A radial artery was cannulated for continuous blood pressure monitoring. $\mathrm{A} \mathrm{CO}_{2}$ pneumoperitoneum was created with an intra-abdominal pressure of $20 \mathrm{mmHg}$ using the da Vinci $^{\mathrm{TM}}$ robot system. A steep Trendelenburg position was maintained to better expose the site to the surgical team. Surgery proceeded at a tidal volume (TV) of 6 $\mathrm{ml} / \mathrm{kg}$ of ideal body weight for a total of $425 \mathrm{ml}$, a respiratory rate $(\mathrm{RR})$ of 12 breaths/min, and a peak inspiratory pressure of $20 \mathrm{cmH}_{2} \mathrm{O} . \mathrm{PetCO}_{2}$ was maintained at $32-35 \mathrm{mmHg}$ with $\mathrm{PaCO}_{2}$ of $35 \mathrm{mmHg}$. 
After the commencement of surgery, $\mathrm{PetCO}_{2}$ gradually increased. One hour after the pneumoperitoneum was created, $\mathrm{PetCO}_{2}$ reached 56 $\mathrm{mmHg}$. Suddenly, the state entropy and response entropy dropped from 36 to 2 and from 34 to 1 , respectively, without any change in the concentration of sevoflurane with a 2 vol. \%. Vital signs were stable with a mean arterial pressure of $70-80 \mathrm{mmHg}$ and an oxygen saturation of 99-100\%. Subsequently, arterial blood gas analysis (ABGA) was checked and revealed severe hypercapnia and respiratory acidosis $\left(\mathrm{pH}: 7.15, \mathrm{PaCO}_{2}\right.$ : $85 \mathrm{mmHg}, \mathrm{PaO}_{2}: 267 \mathrm{mmHg}$ in $\mathrm{FIO}_{2}$ of 0.5 ).

On inspection, subcutaneous emphysema was detected on the patient's anterior chest and neck reaching the patient's mandible. On discussion with the surgeon, intra-abdominal pressure was reduced to 10 $\mathrm{mmHg}$ and the angle of the Trendelenburg position was reduced to $25^{\circ}$. The sevoflurane concentration was reduced to $1-1.5$ vol. $\%$ and the patient was hyperventilated with a TV of $550 \mathrm{ml}$ and an RR of 15 breaths/min. Peak inspiratory pressure was $24 \mathrm{cmH}_{2} \mathrm{O}$ with an $\mathrm{EtCO}_{2}$ of $50-55 \mathrm{mmHg}$. After 30 minutes of hyperventilation, the ABGA showed a slight decrease in $\mathrm{PaCO}_{2}$ and respiratory acidosis $\left(\mathrm{pH}: 7.19, \mathrm{PaCO}_{2}: 75\right.$ $\mathrm{mmHg}, \mathrm{PaO}_{2}: 271 \mathrm{mmHg}$ in $\mathrm{FIO}_{2}$ of 0.5 ). The entropy level stayed at $7-10$. There was no further aggravation of $\mathrm{PaCO}_{2}$ and entropy levels, so the surgeon proceeded with the operation for another hour and hyperventilation was maintained.

After the termination of the operation, the patient was laid in the supine position. $\mathrm{EtCO}_{2}$ dropped to $40 \mathrm{mmHg}$ and entropy immediately recovered to 60 with no change in sevoflurane concentration. Physical examination showed that there was still crepitus on the patient's anterior chest and neck. Out of concern for airway edema, a cuff-leak test was performed. Given that the test showed no air leakage and the patient had a history of having received a cervical spine operation, the patient was sedated with an endotracheal tube for a night in $30^{\circ}$ back-up head-elevated position. The next morning, the patient was fully awake with no neurological deficits and only felt mild crepitus in the anterior chest. The vital signs were stable and ABGA was normal ( $\mathrm{pH}$ : 7.395, $\mathrm{PaCO}_{2}$ : $37.2 \mathrm{mmHg}, \mathrm{PaO}_{2}$ : $267.3 \mathrm{mmHg}$ in $\mathrm{FIO}_{2}$ of 0.5 ). He was extubated the next morning and discharged on postoperative day 10 .

\section{DISCUSSION}

Subcutaneous emphysema was observed to induce hypercapnia followed by a rapid drop in spectral entropy during RALP. Entropy is widely used to track the depth of anesthesia in patients receiving general anesthesia. Other than the concentration of the anesthetic agent, low body temperature, hypotension, hypoglycemia, metabolic derangements, hepatic encephalopathy, and brain injury may influence the level of entropy $[5,6,8]$. When cerebral blood flow (CBF) becomes compromised, changes occur in both the metabolic and electrical activity of cortical neurons which is reflected within seconds by changes in the EEG [9]. Therefore, a drop in entropy levels indicates the depth of anesthesia and can signal other problems associated with cerebral perfusion.

Acute hypercapnia has been reported to induce narcosis and change EEG patterns [7, 10]. Reports suggest that hypercapnia has an anesthetic property with approximately $\mathrm{PaCO}_{2}$ of $137 \mathrm{mmHg}$ equivalent to a 0.5 minimum alveolar concentration in humans [11]. Currently, it is thought that hypercapnia alters gammaaminobutyric acid (GABA) and adenosine levels which inhibits the excitatory synapses in the frontal cortex [12]. Hypercapnia also decreases hippocampal neuronal excitability and causes sedation and anesthesia [13]. Furthermore, during marked hypercapnia with $\mathrm{PaCO}_{2}$ higher than $80 \mathrm{mmHg}$, cerebral resistance vessels are maximally dilated, so cerebral perfusion may be interrupted [14]. This phenomenon would be more pronounced during robot-assisted surgery during which intracranial pressure is already high due to the presence of pneumoperitoneum and a steep Trendelenburg position [2].

Patients who experience a sudden drop in entropy levels caused by acute respiratory acidosis should be promptly inspected for possible causes of the acidosis. When subcutaneous emphysema occurs, the surgeon should be notified and the intra-abdominal pressure should be reduced to halt further aggravation. Hyperventilation should be induced and, if possible, the angle of the Trendelenburg position should be reduced to prevent further increases in intracranial pressure. If ventilation is difficult due to decreased pulmonary compliance or tracheal tube compression from subcutaneous emphysema, immediate conversion to open surgery should occur. Although rare, severe subcutaneous emphysema can lead to fatal complications such as tracheal edema, pneumomediastinum, and pneumothorax [15]. Extubation should be conducted carefully and a cuff leak test or chest X-ray should be conducted for safety. A sudden drop in entropy due to hypercarbia does not present significant risk to the patient if treated promptly $[7,11]$. Anesthesiologists should be aware of and vigilant in monitoring patients for this condition because anesthesia may blunt associated cardiovascular responses [15].

\section{CONCLUSION}

This report presents a case of severe acute hypercapnia which caused reversible depression in entropy during RALP. The narcotic property of $\mathrm{CO}_{2}$ may aggravate sudden drops in entropy.

\section{REFERENCES}

1. Lee JR. Anesthetic considerations for robotic surgery. Korean J Anesthesiol. 2014;66(1):3-11. 
2. Chen K, Wang L, Wang Q, Liu X, Lu Y, Li Y. Effects of pneumoperitoneum and steep Trendelenburg position on cerebral hemodynamics during robotic-assisted laparoscopic radical prostatectomy: A randomized controlled study. Medicine (Baltimore). 2019;98(21):e15794.

3. Gutt CN, Oniu T, Mehrabi A, Schemmer P, Kashfi A, Kraus T. Circulatory and respiratory complications of carbon dioxide insufflation. Dig Surg. 2004;21(2):95-105.

4. Kalmar AF, Foubert L, Hendrickx JF, Mottrie A, Absalom A, Mortier EP. Influence of steep Trendelenburg position and $\mathrm{CO} 2$ pneumoperitoneum on cardiovascular, cerebrovascular, and respiratory homeostasis during robotic prostatectomy. $\mathrm{Br} \mathrm{J}$ Anaesth. 2010;104(4):433-9.

5. Chhabra A, Subramaniam R, Srivastava A, Prabhakar H, Kalaivani M, Paranjape S. Spectral entropy monitoring for adults and children undergoing general anaesthesia. Cochrane Database Syst Rev. 2016;3:CD010135.

6. Taghadomi RJ, Golmakani E, Alizadeh K, Mottahedi B, Rahdari A, Sheybani S. Moderate hypothermia and its effects in reducing the applied dose of anesthetics for patients with opium dependence in cardiac surgery: A randomized controlled trial. Electron Physician. 2016;8(9):2998-3004.

7. Ann J, Jung SM, Park SJ. Decrease in spectral entropy by low tidal volume ventilation-associated severe hypercapnia: a case report. Korean J
Anesthesiol. 2017;70(2):213-6.

8. Vivien B, Langeron $\mathrm{O}$, Riou $\mathrm{B}$. Increase in bispectral index (BIS) while correcting a severe hypoglycemia. Anesth Analg. 2002;95(6):1824-5.

9. Foreman B, Claassen J. Quantitative EEG for the detection of brain ischemia. Crit Care. 2012;16(2):216.

10. Fothergill DM, Hedges D, Morrison JB. Effects of $\mathrm{CO} 2$ and $\mathrm{N} 2$ partial pressures on cognitive and psychomotor performance. Undersea Biomed Res. 1991;18(1):1-19.

11. Yoshida H, Kushikata T, Kabara S, Takase H, Ishihara H, Hirota K. Flat electroencephalogram caused by carbon dioxide pneumoperitoneum. Anesth Analg. 2007;105(6):1749-52, table of contents.

12. Dulla CG, Dobelis P, Pearson T, Frenguelli BG, Staley KJ, Masino SA. Adenosine and ATP link $\mathrm{PCO} 2$ to cortical excitability via $\mathrm{pH}$. Neuron. 2005;48(6):1011-23.

13. Balestrino M, Somjen GG. Concentration of carbon dioxide, interstitial $\mathrm{pH}$ and synaptic transmission in hippocampal formation of the rat. $\mathrm{J}$ Physiol. 1988;396:247-66.

14. Meng L, Gelb AW. Regulation of cerebral autoregulation by carbon dioxide. Anesthesiology. 2015;122(1):196-205.

15. Aldakhil SK, Tashkandi AA, Al Harbi MK, Al Shehri A. Subcutaneous emphysema and hypercarbia as a complication of laparoscopic procedure: case report. J Surg Case Rep. 2020;2020(3):rjz415. 